\title{
Analysis and Research on Basic Model of Hydrogeological Parameters Based on Sensitivity Analysis
}

\author{
Xiaodong Zhang ${ }^{1,2, a^{*}}$, Xiaobo Yang ${ }^{1,2}$, Kangnan Cheng ${ }^{1,2}$, Xin Yue ${ }^{1,2}$, Xixi Liu ${ }^{1,2}$ \\ ${ }^{1}$ Key Laboratory of Salt Lake Resources Exploration in Qaidam Basin, Qinghai Province, Golmud 816000, \\ China \\ ${ }^{2}$ Qaidam Integrated Geological Exploration Institute of Qinghai Province, Golmud 816000, China \\ a 405228726@qq.com \\ *Corresponding author: Xiaodong Zhang
}

Keywords: Sensitivity, Hydrogeological parameters, Model analysis

\begin{abstract}
This paper discusses the sensitivity and uncertainty analysis methods of multi-parameters related to model parameters and model simulation performance. Multi-parameter sensitivity analysis based on Monte Carlo simulation can evaluate the relative importance of multiple parameters in the model. Sensitivity uncertainty analysis can quantitatively evaluate model performance. The case study shows that the number of optimized parameters can be reduced by the sensitivity analysis method. Moreover, before the parameter optimization of the model, the simulation accuracy of the model can be determined based on the sensitivity analysis. For example, the same model can obtain higher simulation accuracy in Chaohe River than in river. The difference of this same model in different watersheds is due to the imperfection of the model structure on the one hand, and the data errors used for modeling on the other hand. The parameter optimization results show that the parameter sensitivity and the overall performance evaluation method of the model are reasonable.
\end{abstract}

\section{Introduction}

From the perspective of the development history of hydrological simulation, hydrological models are all restricted by data, that is, conceptual models are usually established based on the availability of data [1]. The reliability of the conceptual model depends on the appropriateness of the calibration procedure used [2]. Given the data, the key to model calibration is to find a better balance between model complexity and forecast uncertainty. Model calibration algorithms are generally divided into local optimization algorithms and global optimization algorithms. Emery[3] made a detailed summary of these commonly used algorithms. There is no doubt that the global optimum is more convincing than the local optimum. Commonly used global optimization algorithms in the calibration of watershed hydrological models include: simulated annealing, genetic algorithms, and sensitivity analysis [4]. Regarding the evaluation of algorithms, there are mainly two aspects, one is the effectiveness and robustness of the algorithm, and the other is the execution efficiency of the algorithm. Comparative analysis shows that the sensitivity analysis method is more robust and efficient than some commonly used algorithms [5]. While it is important to use appropriate optimization algorithms to determine model parameters, it is also indispensable to evaluate the relative importance of these parameters. For those relatively important parameters, we must be more cautious when determining the parameter value range, because these parameters play a decisive role in the performance of the model. The results of empirical research on hydrological simulation models show that there is a correspondence between sensitivity and importance. It can be seen that sensitivity analysis is a necessary modeling tool because it allows model users to understand the importance of each parameter and the influence of input data errors on the calculation output. Due to the complexity of most hydrological models, it is not feasible to use analytical differentiation to calculate sensitivity. The simple "factor pert urbation" method [6] is a commonly used method in hydrological analysis; among them, the parameter sensitivity is 
generally expressed as the ratio of the output variable to the change value of the parameter. This sensitivity is the absolute sensitivity. It is used for the comparison of parameter sensitivity. Therefore, considering the relative sensitivity of parameter dimension factors is a reasonable method to compare the sensitivity of different parameters in the model. The factor perturbation method analyzes one parameter at a time, and finally synthesizes the results of individual analysis of multiple parameters [7]. This method is generally based on a set of specific parameter values. Obviously, this method does not consider the correlation between the parameters. Multi-parameter Sensitivity Analysis [8] (MPSA) is based on Monte Carlo simulation to change the values of all parameters at the same time, comprehensively considering the results of multiple (such as $N$ times) model runs and simultaneously gives the sensitivity of each parameter. Moreover, the sensitivity measurement is not to compare the change value of the output variable with the change value of the parameter, but to classify the objective function value of the $N$ simulations through the given index according to the defined objective function value (such as the sum of squares of the error), and then calculate the two based on the cumulative frequency of the group, the sensitivity of each parameter is judged. Usually, sensitivity analysis and uncertainty analysis are carried out simultaneously. Uncertainty analysis methods are generally based on more complex Monte Carlo simulations, such as Markov Chain Monte Carlo (MCMC). The MCMC method usually uses algorithms such as Metropolis on the basis of Bayesian theory to sample, so as to obtain the posterior distribution and uncertainty of the parameters. This method can not only evaluate the uncertainty of parameters, but also the uncertainty of model structure and input data.

The advancement of urbanization has caused the proportion of impervious areas in urban areas to continue to increase, and rainfall runoff increases accordingly. In recent years, the problem of urban waterlogging caused by extreme precipitation events such as heavy rains has become increasingly prominent. Due to the randomness and complexity of urban storm runoff and the time-consuming and laborious on-site monitoring, the model has become an important method for studying urban storm runoff pollution management and control. In the process of applying the model, there are two main sources of uncertainty in the model, one is the uncertainty of the model parameters, and the other is the uncertainty of the basic data. The uncertainty of model parameters is because some important hydrological and hydraulic data are difficult to accurately obtain in the process of model establishment, and can only be set according to the empirical values in the own literature or model manual. The basic data mainly includes surface type data, topographic data, remote sensing image data used to determine water catchment area and ground elevation, and drainage network attribute data. The uncertainty of basic data is also because it is difficult to obtain accurately in the actual operation process, even the data that has been obtained will change due to urban construction and transformation.

Parameter sensitivity analysis can test the degree of influence of parameter changes on the model results, and screen out the parameters that have a large impact on the model results and require precise calibration; and for those insensitive parameters that have little effect on the model results, empirical values can be selected. It greatly reduces the workload of model parameter calibration and verification. Research on the parameter sensitivity and stability of the basic model of hydrogeological parameters based on sensitivity analysis is rarely reported. However, due to the sensitivity of the model, the results obtained by different users will be significantly different.

\section{Hydrogeological Parameter Model}

\subsection{Basic Principles}

Over the years, theis equation has played an important role in groundwater hydrology. The graphical method to compare the actual pumping test data with the theoretical curve has become a standard method for determining the hydraulic conductivity and elastic release coefficient of the aquifer[9]. At present, the commonly used solving methods are the standard wiring method and the straight line method (ie, the Jacobian method), but these methods have the disadvantages of heavy workload, more influence by human factors, and non-unique solutions. Based on the principle of the 
least square method and the theis equation as the basic model, this paper introduces the basic principle of using the sensitivity analysis method to calculate hydrogeological parameters. Through practical application, this method is simpler than the current commonly used standard curve wiring method and Jacobian method, economical, and objective.

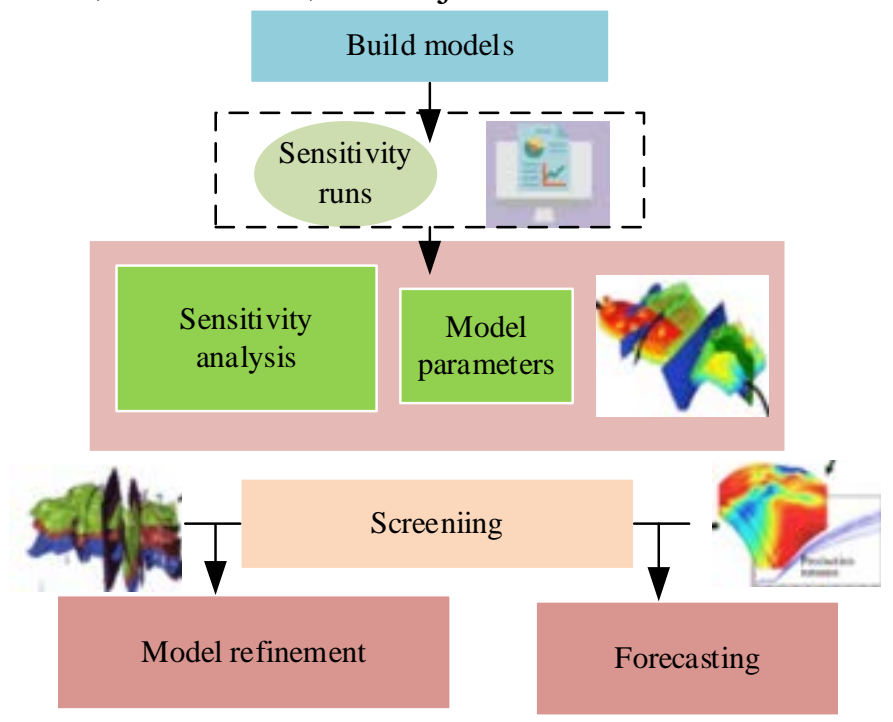

Fig.1 Model Multi-Parameter Sensitivity and Uncertainty Analysis and Parameter Optimization

Because different types of underground aquifers have different calculation formulas for drawdown, their sensitivity coefficients are also different, and they all contain complex generalized integral terms, it is usually difficult to directly solve the value, which limits the application of the sensitivity method. At present, other methods for determining hydrogeological parameters using unsteady flow test data are all applied under the conditions of theis hypothesis. Therefore, this paper applies the basic principles of numerical integration to infer the confined aquifer incomplete wells and several characteristic types of sensitivity coefficient models such as incomplete wells in confined aquifers affected by the unilateral straight line boundary enable the application of the sensitivity method to be smoothly realized on the computer and expand the scope of application[10].

The unsteady flow equation for a complete well of groundwater in a non-leaping confined aquifer is

$$
D=S / 4 \pi t \int_{\lambda r^{2} / 4 t T}^{\infty} \frac{\exp (-\lambda)}{\lambda} d \lambda
$$

In the formula: $t$ is the water conductivity coefficient of the aquifer; $\lambda$ is the elastic release coefficient; $S$ is the pumping flow; $T$ is the pumping time, calculated from the beginning of the pumping; $r$ is the distance between the observation hole and the center of the pumping well.

\subsection{Depth and Sensitivity Analysis Models of Various Feature Types}

Multi-parameter sensitivity analysis includes the following steps:

(1) Select test parameters; (2) Set the value range of each parameter according to the measured values of the field and indoor experiments; (3) For each selected parameter, generate a sequence, such as generating $N$ within the value range uniformly distributed independent random numbers; (4) Apply the generated $N$ random numbers to run the model, and calculate the corresponding objective function value; (5) Compare the objective function value with the given index (R) to determine $N$ parameters among the values, which are "acceptable" and which are "unacceptable"; (6) Evaluation parameter sensitivity: for each parameter, compare the distribution of the two sets of parameter values "acceptable" and "unacceptable" (calculate the cumulative frequency and draw the cumulative frequency curve). If the two distributions are the same, it means that the parameter is not sensitive, otherwise, the parameter is more sensitive. The degree of separation of the two cumulative frequency curves represents the sensitivity of the parameter, and the "objective function 
value" is represented by the sum of squares of the error between the simulated value and the measured value. The "measured value" is usually obtained by substituting the median value of each parameter value range into the model for simulation, rather than the actual observation value in the usual sense[8]. The value range (minimum and maximum) of each parameter is determined by parameter estimation and field measurement of the study area. If the simulated objective function value is less than the "subjective index", the result is considered "acceptable"; otherwise, the result is considered "unacceptable".

Different types of aquifers have different depth calculation models and different sensitivity coefficient models. Because the models contain complex generalized integral terms, it is necessary to derive their respective models and their solution methods in advance before they can be iteratively calculated from the computer Solve the best hydraulic conductivity and elastic water release coefficient. According to the principle of numerical integration, this article has derived various calculation models. Due to space limitations, only the results of some of these models are listed here.

The sensitivity coefficient model is:

$$
\begin{gathered}
D=S / 4 \pi t \delta(\lambda) \\
\delta(\lambda)=-0.6-\ln (\lambda)-\sum_{i=1}^{n}(-1)^{i} \lambda^{i} /(i \times i !) \\
\lambda=\lambda^{*} r^{2} / 4 t T
\end{gathered}
$$

\section{Example Application}

\subsection{Knowledge Acquisition and Cleaning}

The distance between the observation hole and the pumping hole is $140 \mathrm{~m}$, and the pumping flow rate is $60 \mathrm{~m}^{3} / \mathrm{h}$. The study area of this paper is the Chaobai River Basin above Miyun Reservoir in North China, with an area of $13,846 \mathrm{~km}^{2}$ and divided into 136 sub-basins. 10 years of hydrometeorological data are used for case studies. The hydrological model uses a monthly water balance model. Since the focus of this article is to discuss multi-parameter sensitivity and uncertainty analysis, it is applicable to any model. The parameters of the model are shown in Table 1.In this paper, the observation records of a single well pumping test are shown in Table 2.

Table 1 Parameters to Be Optimized in the Model

\begin{tabular}{|l|l|l|}
\hline Parameter meaning & Lower limit of value & Upper limit of value \\
\hline Surface runoff coefficient & 0.04 & 0.42 \\
\hline Surface Runoff Index & 0.08 & 3.02 \\
\hline Soil water storage and discharge coefficient & 0.04 & 0.12 \\
\hline Evapotranspiration calculation parameters & 9.10 & 1.02 \\
\hline Initial value of soil moisture & 5.00 & 30.00 \\
\hline
\end{tabular}

Table 2 Observation Data of Single Well Pumping Test

\begin{tabular}{|l|l|l|}
\hline Number of observations & Cumulative time (min) & Depth of observation well $(\mathrm{m})$ \\
\hline 1 & 20 & 0.48 \\
\hline 2 & 40 & 0.66 \\
\hline 3 & 60 & 1.02 \\
\hline 4 & 80 & 1.12 \\
\hline 5 & 100 & 1.34 \\
\hline 6 & 120 & 1.46 \\
\hline 7 & 140 & 1.51 \\
\hline 8 & 160 & 1.73 \\
\hline
\end{tabular}

According to the basic principle of the sensitivity analysis method, the calculation program is 
compiled, and the iteration accuracy of $\mathrm{t}$ and $\lambda$ are set to 0.01 and 0.00005 respectively. The calculation results are shown in Table 3. At the same time, Table 2 also lists the calculation results of the standard curve wiring method and the Jacobian method for comparison. The water level fitting curve calculated by the sensitivity method is shown in Figure 2.

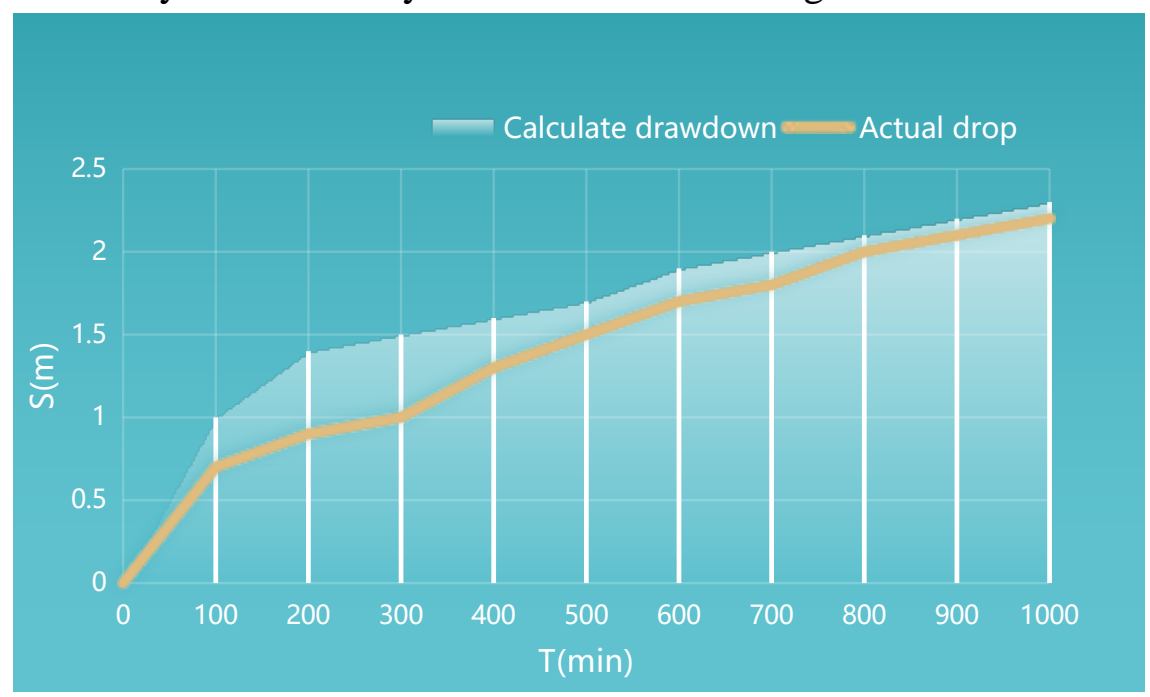

Fig.2 The Water Level Fitting Curve of the Calculation Result of the Sensitivity Method of the Pumping Test

Table 3 Comparison of Calculation Results

\begin{tabular}{|l|l|l|l|}
\hline & Sensitivity analysis & Standard curve method & Jacob Method \\
\hline $\mathrm{T}\left(\mathrm{m}^{2} / \mathrm{d}\right)$ & 189.34 & 195.47 & 193.35 \\
\hline$\lambda$ & $2.13 \mathrm{e}-4$ & $2.47 \mathrm{e}-4$ & $2.39 \mathrm{e}-4$ \\
\hline
\end{tabular}

It can be seen from Table 2 that the calculation results of the sensitivity analysis method are very consistent with those of the other two conventional methods. Judging from the fitting curve of the calculated and measured drawdown in Figure 1, the fitting result is very good.

\section{Conclusion}

Through theoretical derivation and practical application, it is shown that the sensitivity analysis method to determine hydrogeological parameters is feasible. Compared with the wiring method and the straight line method, it is simple, practical, accurate and objective, and is not affected by human factors. Using computer programs, the solution process can be completed quickly. Solving the problem of determining hydrogeological parameters under the conditions of theis hypothesis by solving the calculation models of multiple characteristic types of depression and sensitivity coefficients, and exploring a method for determining hydrogeological parameters by fully considering various stratigraphic conditions in the future.

\section{References}

[1] Borgonovo, Emanuele, et al. "Making the Most out of a Hydrological Model Data Set: Sensitivity Analyses to Open the Model Black - box.” Water Resources Research,vol.53, no.9, pp.7933-7950,2017.

[2] Mockler, Eva M., Fiachra E. O’Loughlin, and Michael Bruen. "Understanding hydrological flow paths in conceptual catchment models using uncertainty and sensitivity analysis." Computers \& Geosciences,vol. 9, no.1, pp.66-77,2016.

[3] Emery, Charlotte M., et al. “Temporal Variance-based Sensitivity Analysis of the River-routing Component of the Large-scale Hydrological Model ISBA-TRIP: Application on the Amazon Basin.” Journal of Hydrometeorology,vol. 17,no.12, pp.3007-3027,2016. 
[4] Khalid, K., et al. "Application on One-at-a-time Ssensitivity Analysis of Semi-distributed Hydrological Model in Tropical Watershed.” International Journal of Engineering and Technology, vol.8, no.2, pp.132-136,2016.

[5] Welde, Kidane, and Bogale Gebremariam. "Effect of Land Use Land Cover Dynamics on Hydrological Response of Watershed: Case study of Tekeze Dam Watershed, Northern Ethiopia.” International Soil and Water Conservation Research,vol. 5,no.1, pp.1-16,2017.

[6] Zhang, Junlong, et al. “Assessment of Parameter Uncertainty in Hydrological Model Using a Markov-Chain-Monte-Carlo-based Multilevel-Factorial-Analysis Method.” Journal of Hydrology, vol.53,no.8, pp.471-486,2016.

[7] Del Giudice, Giuseppe, and Roberta Padulano. "Sensitivity Analysis and Calibration of a Rainfall-runoff Model with the Combined use of EPA-SWMM and Genetic Algorithm." Acta Geophysica, vol.64, no.5, pp.1755-1778,2016.

[8] Unice, K. M., et al. "Characterizing Export of Land-based Microplastics to the Estuary-Part II: Sensitivity Analysis of an Integrated Geospatial Microplastic Transport Modeling Assessment of Tire and Road Wear Particles.” Science of the Total Environment,vol. 6, no.4, pp.1650-1659,2019.

[9] Mahrez, Boulabeiz, et al. "GIS-based GALDIT Method for Vulnerability Assessment to Seawater Intrusion of the Quaternary Coastal Collo Aquifer (NE-Algeria).” Arabian Journal of Geosciences, vol.11, no.4, pp.71-72,2018.

[10] Maina, Fadji Zaouna, and Alberto Guadagnini. "Uncertainty Quantification and Global Sensitivity Analysis of Subsurface Flow Parameters to Gravimetric Variations during Pumping Tests in Unconfined Aquifers.” Water Resources Research,vol. 54, no.1,pp. 501-518,2018. 